\title{
Droplet Dynamics on Nanostructured Doubly Reentrant Surfaces
}

\author{
Dong Liao, Huihe Qiu \\ Department of Mechanical and Aerospace Engineering \\ The Hong Kong University of Science and Technology \\ Clear Water Bay, Kowloon, Hong Kong \\ dliao@connect.ust.hk; meqiu@ust.hk
}

\begin{abstract}
A nanostructured surface is proposed for potential application of anti-icing. This surface integrates microcavities with doubly reentrant nanostructures which can simultaneously enhance static repellency and dynamic pressure resistance. This surface is inspired by the skin structure of a spring tail which can survive even in oil and ethenal. We fabricated nanostructures with overhanging edge which exhibited great wetting resistance ability. It is found that when a droplet impacting on different surfaces, the newly fabricated nanostructured doubly reentrant surface shows a shortest contact duration in comparison with other surfaces. Therefore, the nucleation for icing on a cold doubly reentrant nanostructures is depressed and the surface shows icephobic property. Our new structure showed great wetting resistance ability in both static and dynamic conditions, under low temperature and the theoretical analysis accorded with the existing simulation work. The decrease of contact time for the new surface also gave the potential in applications like anti-icing in the future.
\end{abstract}

Keywords: Nanostructure, Doubly reentrant, Closed cavity, Droplet impact, Icephobic, Superomniphobic.

\section{Introduction}

Superomniphobic surfaces are commonly observed in nature and have wide applications. Inspired by the skin structure of a spring tail which can survive even in oil and ethenal ${ }^{1}$, structures with overhanging edge were provided recently and exhibited great wetting resistance ability ${ }^{2,3}$. Among these new surfaces, doubly re-entrant structure showed highest hydrophobicity cause it, for the first time, successfully repelled FC-72 ${ }^{4}$. However, dynamic studies and studies in freezing temperature still lack. In our work, visualization techniques of high-speed camera, droplet generating system and cooling chamber were applied to capture the details of the transient droplet impacting process on the new surface. Based on which the impacting performance was studied, and the impacting time was also measured and compared. The new structure showed great wetting resistance ability in both static and dynamic conditions, and the theoretical analysis accorded with the existing simulation work 5 .

\section{Experimental Methods}

The experiment setup for contact angle measurement and droplet impact was established as shown in Figure 1(a). The LED lamp provided cold light and the high-speed camera caught the images of droplet dynamic process. Sample plate was set in center, above which was the adjustable-height needle connecting to the threaded syringe, which can precisely control the droplet volume within $0.1 \mu \mathrm{L}$, to ensure smoothly change of the droplet volume during experiments. Since the impacting process was so fast, both time interval and exposure time should be small enough. Detailed parameters of the high-speed camera setting in the experiment are listed in Table 1.

Table 1: Setting information of high-speed camera.

\begin{tabular}{cccc}
\hline & FPS & Resolution & Exposure $(\mu s)$ \\
\hline CA Measurement & 50 & $1280^{*} 960$ & 1995 \\
Droplet Impact & 5000 & $640^{*} 480$ & 16 \\
\hline
\end{tabular}


The cooling chamber was removable and set at the same place as the sample plate with its structure illustrated in Figure 1(b). The wall of the cooling chamber was made by aluminum with the front and back being transparent, around which were the thermoelectric coolers connected by the water-cooling modules. The outer layer of the chamber was made from Styrofoam to reduce heat loss. Another thermoelectric cooler module was mounted under the test chamber to change the temperature of the substrate. Two pipes were connected to the test chamber to control humidity. Two temperature sensors were used to measure the substrate and air temperature respectively. One humidity sensor was also applied inside. A droplet generator was mounted at the top of the chamber.

(a)

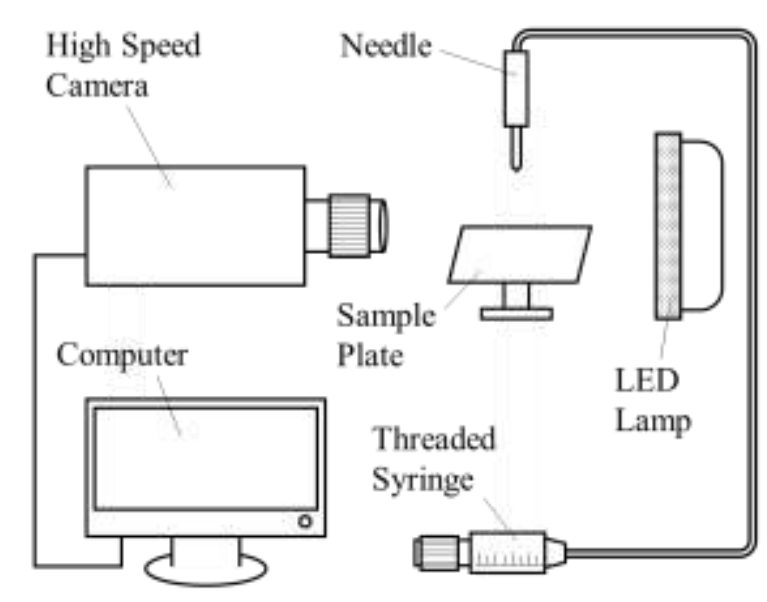

(b)

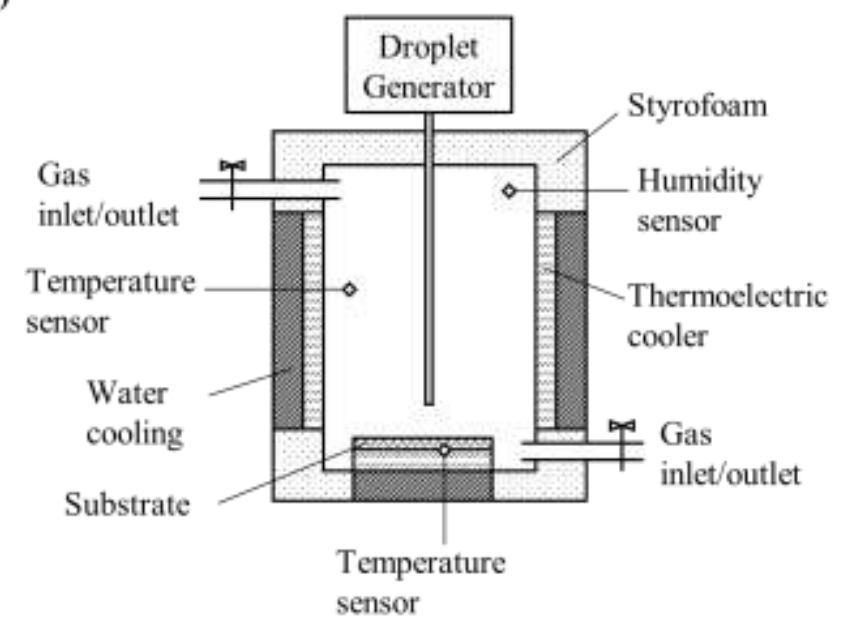

Fig. 1: (a) The schematic of experiment setup; (b) The structure of the cooling chamber.

\section{Results and Discussion}

For static contact angle measurement, the microstructure of the surface is shown in Figure 2(a). Without any surface treatment methods, both water and FC-40 achieved high contact angle as illustrated in Figure 2(b). However, the structure of this surface was relatively large, for the pitch distance was over $100 \mu \mathrm{m}$. Since the small droplets can be smaller than $30 \mu \mathrm{m}$ in real applications, we then fabricated a new surface as illustrated as surface A in Figure 2(c). Also, a sample without overhanging edge was fabricated to be compared as the traditional closed-cell structure surface, which was regarded as the best deign ${ }^{6}$ for droplet rebounding as shown in Figure 2(c) named surface B. Detailed parameters are listed in Table 2. Before doing experiments, both surface A and B were coated with FAS $(1 \mathrm{H}, 1 \mathrm{H}, 2 \mathrm{H}, 2 \mathrm{H}$-Perfluorodecyltriethoxysilane).

Figure 2(d) illustrated the details of droplet impacting. The droplet firstly impacted on the substrate and a thin layer water formed at the bottom. Later, the edge of this layer expanded to the largest and then went back to center. Some small 
droplets splashed at the edge when the impacting velocity reached a critical value. And the main part of the droplet was then rebounded from the surface if it was not wetted, otherwise the droplet would stick on it. Since the time interval of the images was constant, the contact time can also be measured and compared.

(a)

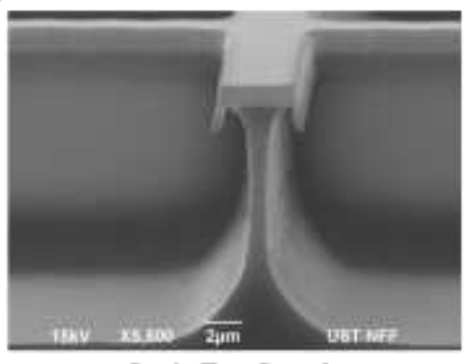

Static Test Sample

(b)

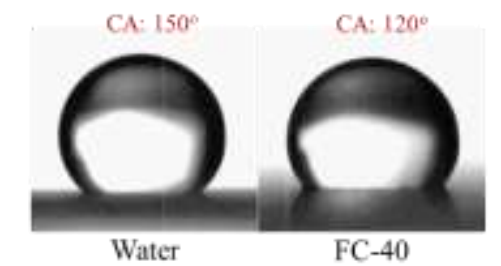

(c)

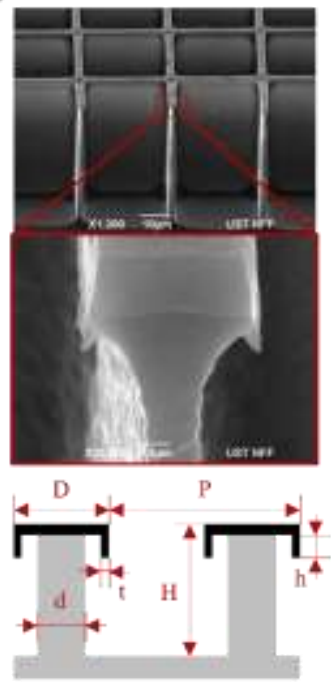

Surface A
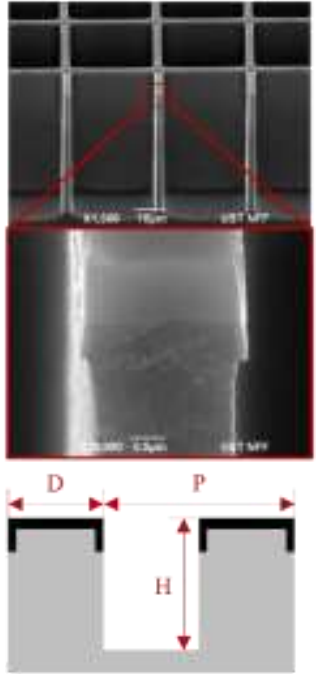

Surface B

(d)

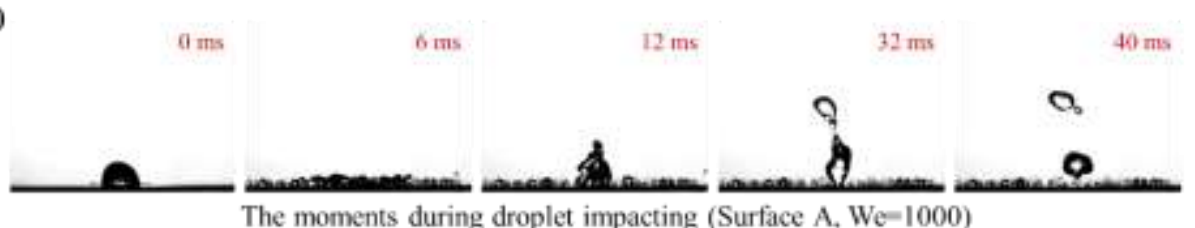

Fig. 2: (a) The micro structure of the static test sample; (b) Contact angle of water (surface tension: $72 \mathrm{mN} / \mathrm{m}$ ) and FC-40 (surface tension: $16 \mathrm{mN} / \mathrm{m}$ ) on the static test sample; (c) Micro structure of surface A and B; (d) The moments during the droplet impacting (surface $\mathrm{A}, \mathrm{We}=1000$ ).

Table 2: Parameters of the micro structure for surface A and B.

\begin{tabular}{cccccccc}
\hline & $\mathrm{D}(\mu \mathrm{m})$ & $\mathrm{P}(\mu \mathrm{m})$ & $\mathrm{H}(\mu \mathrm{m})$ & $\mathrm{d}(\mu \mathrm{m})$ & $\mathrm{t}(\mathrm{nm})$ & $\mathrm{h}(\mu \mathrm{m})$ & Solid Fraction \\
\hline Surface A & 2.5 & 30 & 35 & 0.5 & 100 & 0.8 & 0.16 \\
Surface B & 2.5 & 30 & 35 & $/$ & $/$ & $/$ & 0.16 \\
\hline
\end{tabular}

In dynamic studies, both surface A and B successfully rebounded droplets under high Webber number conditions as illustrated in Figure 3(a), while surface A showed better rebound ability in both small and large Webber number conditions. With the assistance of high-speed camera, the contact time during the droplet impacting process was measured for it strongly affected the heat transfer between the droplet and substrate as shown in Figure 3(b), and it is clear that our new surface had shorter contact time compared with the traditional surface. Theoretical analysis of Laplace breakthrough and Canthotaxis effect was conducted to analyze the micro wetting behavior at the top, and the values are $100.4 \mathrm{hPa}$ and $20 \mathrm{hPa}$ for surface A and $\mathrm{B}$, respectively; adiabatic compression model was used, noted as 'air-spring effect', to describe the pressure change inside the cavities, which is around $496 \mathrm{hPa}$ when compressed to be $3 / 4$ as the initial volume. Based on the existing simulation work, the peak value as illustrated in figure 3(c) of the pressure distribution is estimated to be around $400 \mathrm{hPa}$ when We equals to 1100 , which accorded with the value of adiabatic compression. 


\section{Conclusion}

Air-spring effect plays the main role in high-pressure region, while in low-pressure region the level of breakthrough pressure at top differ the wetting resistance of our new surface to the traditional ones. The two mechanisms work together to render our new surface with higher wetting resistance ability and reduce the contact time. Under freezing temperature, ice nucleation usually happens when the contact time exceed a critical value. Thus, reduced contact time also gives enhanced icephobicity of the new surface. We believe this work will be helpful in designing novel robust superomniphobic surfaces in applications like aircraft anti-icing and prolonged biofluid resistance.

(a)

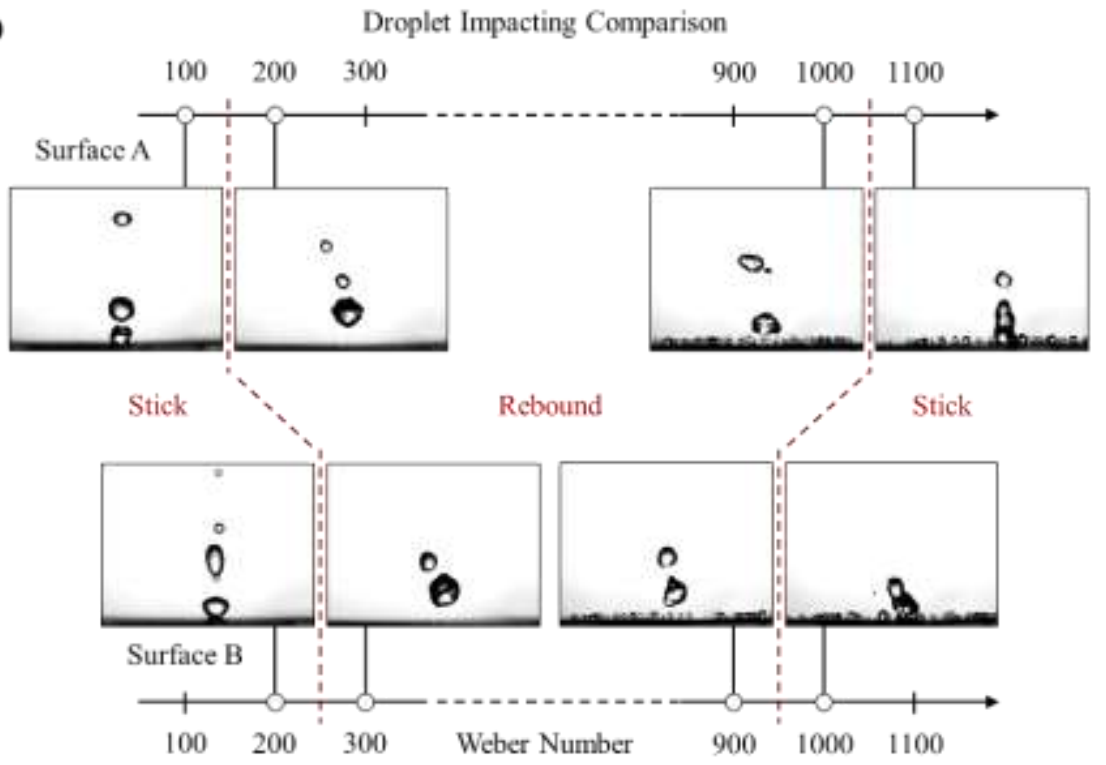

(b)

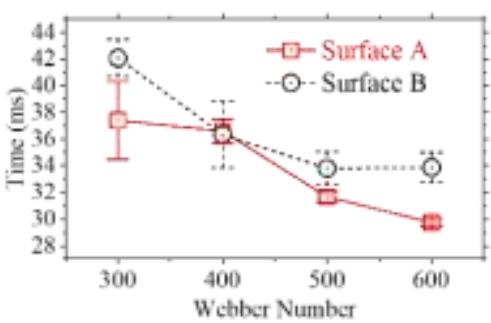

(c)

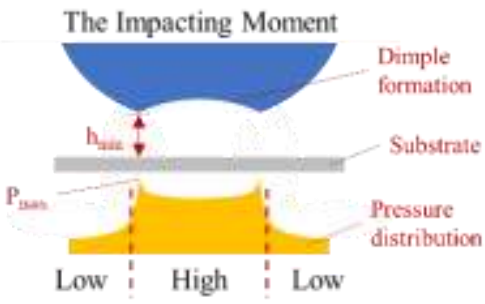

Fig. 3: (a) Droplet rebound conditions under different Webber numbers; (b) Contact time comparison for surface A and B; (c) Pressure distribution on the substrate at the moment of droplet impacting.

\section{Acknowledgements}

This work was supported by the Research Grants Council of the Government of Hong Kong Special Administrative Region (HKSAR) with RGC/GRF Project No. 16207515.

\section{References}

[1] R. Hensel, R. Helbig, and S. Aland, A. Voigt, C. Neinhuis and C. Werner, "Tunable nano-replication to explore the omniphobic characteristics of springtail skin," NPG Asia Materials, vol. 5, no. 2, pp. e37, 2013.

[2] J. Choi, W. Jo, S.Y. Lee, Y. S. Jung, S. H. Kim, H. T. Kim, "Flexible and robust superomniphobic surfaces created by localized photofluidization of azopolymer pillars," ACS Nano. vol. 11, pp. 7821-7828, 2017.

[3] A. Tuteja, W. Choi, J. M. Mabry, G. H. McKinley, and R. E. Cohen, "Robust omniphobic surfaces," Proceedings of the National Academy of Sciences, vol. 105, no. 47, pp. 18200-18205, 2008. 
[4] T. Liu, C. J. Kim, “Turning a surface superrepellent even to completely wetting liquids,” Science, vol. 346, no. 6213, 2014.

[5] M. Mani, S. Mandre, M. P. Brenner, "Events before droplet splashing on a solid surface," Journal of Fluid Mechanics, no. 647, pp. 163-185, 2010.

[6] L. Mishchenko, B. Hatton, V. Bahadur, J. A. Taylor, T. Krupenkin, J. Aizenberg, "Design of Ice-free Nanostructured Surfaces Based on Repulsion of Impacting Water Droplets," ACS Nano., vol. 4, pp. 7699-7707, 2010 . 